\title{
Transmission EELS Quantification Study of Oxygen Content at Zirconium Alloy Metal/Oxide Interface
}

\author{
Na Ni, Sergio Lozano-Perez, John Sykes, Chris Grovenor
}

Department of Materials, Oxford University, Parks Road, Oxford, OX1 3PH, UK

A great deal of careful experimentation has been carried out over the past 40 years on the critical mechanisms of oxidation in the zirconium alloys used for nuclear fuel cladding. However, there is still no consensus on how the nano-scale structure and chemistry of the metal/scale interface influence the oxidation kinetics, although this information is vital for the accurate prediction of the performance of existing alloys and the design of new materials for higher burn-up regimes. The presence of an oxygen-rich region at the metal/oxide interface has been previously reported as a suboxide phase [1-3]. Since oxidation of $\mathrm{Zr}$ alloys proceeds primarily by inward diffusion of oxidizing species, such a sub-oxide could play a crucial role in controlling oxidation kinetics, but there is still considerable disagreement on the precise composition and structure of this layer. Previous quantitative analysis on the metal/oxide interface were all carried out by EDX, which is not ideal for the accurate analysis of light elements such as oxygen. In this work we show that reliable quantification results on the oxygen content at the metal/oxide interface can be obtained by electron energy-loss spectroscopy (EELS) analysis when employing the most up-to-date TEM sample preparation and analysis techniques.

TEM thin foils were prepared from oxides on Zirconium alloys by employing the in situ lift-out FIB method $[4,5]$ on a FEI FIB 200 instrument $\left(5-30 \mathrm{kV} \mathrm{Ga}^{+}\right.$incident beam energy with currents of 50$5000 \mathrm{pA}$ ). This technique has the advantage of producing large, homogenous, electron transparent foils less than $100 \mathrm{~nm}$ thick containing the metal/oxide interface as required for EELS. TEM and EELS analysis were carried out on a Jeol 3000F microscope, equipped with a Gatan GIF and operated at $297 \mathrm{kV}$.

A series of calibrations were necessary to achieve reliable quantitative EELS data. First, a new experimental ratio between $\mathrm{Zr}$ and $\mathrm{O}$ inelastic scattering cross-sections $\left(\sigma_{\mathrm{zr}} / \sigma_{\mathrm{o}} \approx 7.27\right)$ was obtained using a stoichiometric $\mathrm{ZrO}_{2}$ powder sample. After this calibration, the measured oxygen content in the bulk oxide was found to be very close to the 67 at. \% theoretical oxygen content of $\mathrm{ZrO}_{2}$, compared to $\sim 80$ at. \% before calibration. Secondly, to evaluate the influence of multiple scattering, the inelastic mean free path, $\lambda$, under our experimental conditions $(\alpha=11.5 \mathrm{mrad}$ and $\beta=9 \mathrm{mrad})$ was estimated by acquiring a series of EELS spectra from a specially prepared wedge-shaped TEM specimen ( $1^{\circ}$ angle) (Figure 1$)$. The absolute thickness was determined from a HAADF line profile along the line shown in Figure $1 \mathrm{~b}$. A linear fitting of $t / \lambda$ vs. $t$ yielded an experimental value of $\sim 99 \mathrm{~nm}$ for $\lambda$, significantly smaller than the calculated value of $115 \mathrm{~nm}$ [6]. Quantitative results from the wedge-shaped sample confirmed that the expected $\mathrm{Zr} / \mathrm{O}$ ratio for the $\mathrm{ZrO}_{2}$ far from the interface was obtained for sample thicknesses below 80nm.

A commercial Zircaloy-4 recrystallized sheet $(1.24 \% \mathrm{Sn}, 0.17 \% \mathrm{Fe}, 0.1 \% \mathrm{Cr}$, w/w) tested in an autoclave at $360{ }^{\circ} \mathrm{C}$ for 54 days developed a $1.2-1.5 \mu \mathrm{m}$ pre-transition oxide and was characterized using our new methodology. The HAADF image in Figure 2a shows a distinct layer of intermediate contrast $\sim 50 \mathrm{~nm}$ thick between the metal and the columnar oxide. The EELS line profile (figure $2 \mathrm{~b}$ ) 
across the same interface reveals an intermediate suboxide layer of similar thickness with a relatively constant oxygen content of $\sim 45 \%$ (as opposed to the $\sim 66 \%$ oxygen measured in the bulk oxide). We are using this methodology to explore whether these suboxide layers may explain some of the observed differences in corrosion performance between different $\mathrm{Zr}$-alloys.

References

[1] A. Yilmazbayhan et al., Journal of Nuclear Materials 349 (2006) 265.

[2] P. Bossis et al, Zirconium in the Nuclear Industry: 12th International Symposium, 2000, 918.

[3] S. Abolhassania et al., Journal of Nuclear Materials Article in Press (2009).

[4] J. Mayer et al., MRs Bulletin 32 (2007) 400.

[5] S. Lozano-Perez, Micron 39 (2008) 320.

[6] T Malis et al., Journal of Electron Microscopy Technique 8 (1988) 193.

[7] The authors would like to thank their collaborators from EDF Energy, Westinghouse, and Open and Manchester Universities that make up the MUZIC consortium. This research was funded by the Engineering and Physical Sciences Research Council (EPSRC) under grant number EP/E036384/1.
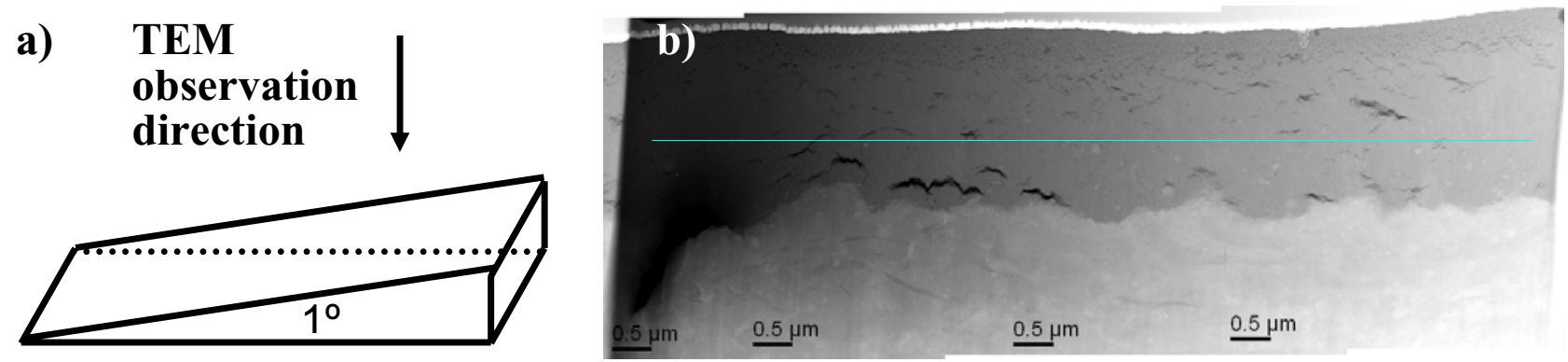

Figure 1: a) Schematics of the FIB prepared wedge sample. b) HAADF image of the wedge sample.
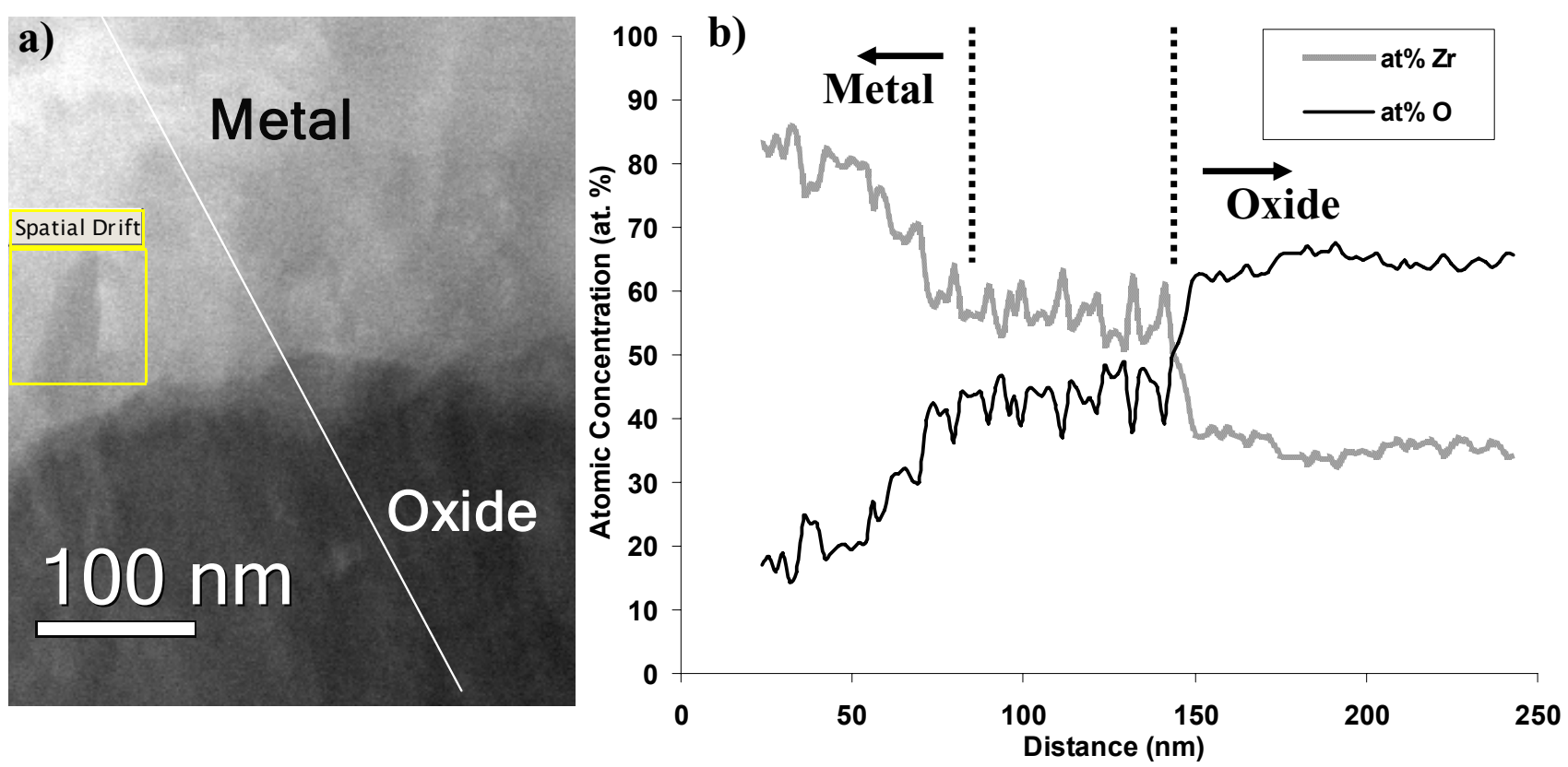

Figure 2 a) HAADF image of the metal/oxide interface region and b) oxygen concentration line profile across the metal/oxide interface showing the presence of a sub-stoichiometric oxide layer. 